\section{Geophysics of Mars}

\section{Garry E. Hunt}

Geophysics of Mars. Developments in Solar System and Space Science, Vol.4. By R. A. Wells. Pp.678. (Elsevier Scientific: Amsterdam, Oxford and New York, 1979.) \$87.75; Dfl. 180.

FOR centuries, the planet Mars has been the centre of intrigue for Mankind. Across the cold icy darkness of space, there has always been speculation that life could have developed on our planetary neighbour. In recent years, detailed observations from spacecraft have added to our measurements of the Martian environment by Earth-based telescopes, so that we are now building a fairly comprehensive picture of the planet now, and some interesting indications of its past. In this monograph, Dr Wells has produced a huge text which tries to put into perspective our current understanding of the geophysics of Mars. I believe he has largely succeeded in a well written and carefully illustrated text.

Naturally, the structure of the text and its content must have some personal biases, but fortunately all of the eight chapters have a wealth of references to supplement the material, so that the reader will not feel that his understanding of Mars is too strongly influenced by the author's own views. We are led into the discussions of Mars through the physical interaction of the atmosphere and surface. The importance of the Martian atmosphere in shaping some of the surface features and the minor constituents in providing information on the past history of the planet are reviewed. The chapter continues by introducing the basic spectroscopic matters related to the detection of atmospheric constituents such as $\mathrm{CO}_{2}, \mathrm{CO}$, $\mathrm{O}_{3}$ and $\mathrm{H}_{2} \mathrm{O}$, their variability, and the basic techniques of determining the surface pressure and aerosol content of the atmosphere. Mars does possess a very exciting meteorology and I personally think it is unfortunate that this characteristic has not been highlighted more. There is some discussion of the cloud systems seen on the planet, but those seen by Viking in the Tharsis area, such as bore wave clouds, are unfortunately incomplete. The discussion of the dust storm generation misses out the fundamental observations by the Viking imaging and infrared instruments which have clearly shown that separate mechanisms are responsible for the preperihelion and perihelion storms.

Two of the most controversial aspects of Mars are concerned with the obscuration of the dark surface markings at short wavelengths and the progressive, seasonal, cyclic increase in their darkness at long wavelengths. These aspects are more familiarly known as the blue haze and the seasonal wave of darkening, respectively. A complete chapter is devoted to these matters. The discussions are very thorough. The reader will also be introduced to some of the problems and applications of Mie scattering theory in a simple and effective way which is always useful.

The next three chapters deal with different aspects of the basic surface properties of the planet. The geological aspects of Mars are built up from our early telescopic observations and subsequently improved with the spacecraft data through the 1960 s until the present Viking data is available. A brief discussion is given of the problem of estimating the age of a surface by crater counting, but I am not sure whether the reader will necessarily know which set of results he should believe at this stage. The remaining two chapters of this sequence cover the Martian topography and the linear features. It may be sad for some readers that Lowell's infamous canals do not relate to any of the Martian features. However, this situation is in itself a valuable calibration, and a direct indication of the care we must take in interpreting visual observations.

Two further chapters cover the internal structure of the planet, mantle convection and the Martian moons, Phobos and Deimos. The monograph concludes with a detailed discussion of the first few months of the Viking view of Mars. As a result, a great deal of the seasonal aspects, and all the annual variations of the atmosphere, are not included.

This book has been written with great care, and there is every indication that the author has devoted himself to this task of placing on record the geophysics of Mars. I certainly believe that it is a valuable addition to the discussion of the Martian environment, and is well supplemented by a large number of high quality diagrams and photographs. But how many people, or indeed libraries, can really afford $f 41$ ? I wish publishers would try to be a little more realistic in their prices; then good books, such as this one, would reach a much bigger audience.

Garry E. Hunt is Head of the Laboratory for Planetary Atmospheres, Department of Physics and Astronomy, University College, London, $U K$

\section{Electron microscopical techniques}

\section{S.J. Kirchanski}

Electron Microscopy and Cytochemistry of Plant Cells. By J.L. Hall. Pp.444. (Elsevier/North Holland: Amsterdam and New York, 1979.) \$46.25; Dfl.95.

THIS book is indeed a pleasant surprise; all too often edited volumes are very uneven in quality and contain unfocused or inappropriate chapters. On the contrary, in this work all the chapters are of good quality and contribute an overall unified aspect to the volume. There are seven chapters which cover preparation of thin sections, enzyme cytochemistry, labelled antibodies and lectins, ion localization, autoradiography, freeze fracture and scanning electron microscopy.
Each chapter begins with a useful outline of its contents. The reader is introduced to the techniques covered in the particular chapter, and in some cases a brief historical review of the subject is undertaken. This is followed by detailed descriptions of the various technical steps along with informative discussions of alternate methods in most cases. Each chapter is thoroughly illustrated and has a good, current bibliography. The overall quality of printing is high and the micrographs are well reproduced. The chapters on thin sections, cytochemistry and lectins each end with appendices of methods and recipes which will doubtless prove extremely popular with most researchers.

Of course, given the space restraints of the volume, it is not possible for each chapter to be absolutely complete. Different workers will undoubtedly notice different omissions. In the chapter on thin sectioning I was pleased by the detailed treatment of staining (although I believe that Reynolds should have been credited with the lead citrate procedure that was presented), but I felt that insufficient discussion was given to the value of low viscosity resins for embedding plant materials. In the same way, though I felt that the historical treatment of freeze fracture and the descriptions of standard freeze-fracture methods were masterful, I think that insufficient coverage was given to low-temperature, rapid freezing (Heuser-liquid helium methods) and the revolution that rapid freezing is currently causing in freeze fracture. Nevertheless, this is an excellent volume and should be available in all biological research libraries. Although the material is aimed towards plant biologists, it is of use and value to all biological electron microscopists. In spite of the volume's rather high price, I predict that many researchers will purchase it as a personal reference book.

S.J. Kirchanski is a Postdoctoral Research Fellow and Lecturer at Harvard University Biological Laboratories, Cambridge, Massachusetts. 